\title{
Electrical Recording Thermometers for Clinical Work
}

This content has been downloaded from IOPscience. Please scroll down to see the full text. 1909 Proc. Phys. Soc. London 22220

(http://iopscience.iop.org/1478-7814/22/1/317)

View the table of contents for this issue, or go to the journal homepage for more

Download details:

IP Address: 137.132.123.69

This content was downloaded on 03/10/2015 at 08:27

Please note that terms and conditions apply. 
XVI. Electrical Recording 7 hermometers for Clinical Work. $B y$ H. L. Callendar, M.A., LL.D., F.R.S., Professor of Physics at the Imperial College of Science and Technology, S. $W{ }^{*}$

In Memoriam Dr. A. GAMGEE, F.R.S., obiit Mar. 29, 1909.

1. THE scientific interest and importance of a method of continuously recording the temperature of the body, whether in health or disease, is now being generally recognized, and I have been persuaded that my experiments in the construction and testing of suitable apparatus for the purpose may be of some value at the present juncture, although I have no results of physiological importance to produce.

My attention was first directed to the subject by Professor J. G. Adami, F.R.S., of McGill College, Montreal, at whose suggestion I constructed some electrical resistance thermometers specially adapted for recording the temperature of different parts of the suface of the body. Unfortunately I had to leave Montreal early in 1898 , before the apparatus had taken its final shape, and the results obtained at that time, consisting merely of experimental records of my own normal temperature, did not seem to be of sufficient interest to merit publication. I did not return to the subject until Norember 1908, when I undertook to make some thermometers for Dr. A. Gamgee, F.R.S., and to collaborate with him in evolving a practical method suitable for general use in clinical work. His paper, then recently published, "On Methods for the Continuous (Photographic) and Quasi-Continuous Registration of the Diurnal Curve of the T'emperature of the Animal Body" $\dagger$, dealt exclusively with thermoelectric methods of temperature measurement: but he had become eonvinced that the thermoelectric method was unsuitable for general use, on account of the delicacy of the apparatus, and tho necessity for employing an elaborate thermostat. The Copper-Constantan thermocouples which he employed gave an E.M.F. of 40 microvolts per $1^{\circ} \mathrm{C}$., which was ample for delicate photographic methods of registration, but insufficient

* Presidential Address, February 11, 1010.

† 1'hil. Trans. Roy. Soc. J3. vol. 200, p. 219 (1406). 
for the more robust type of instrument required for general use with ink records. A more serious difficulty in practice was the necessity of keeping one of the junctions of the couple at a constant temperature in the neighbourhood of $37^{\circ} \mathrm{C}$. for long periods. In the application of the resistance method no thermostat was required, and it was easy to obtain a tenfold larger deflexion with the same galvanometer.

The objections that have commonly been urged against electrical resistance thermometers for this kind of work are (1) that resistance thermometers are more difficult to construct and to insulate satisfactorily than thermocouples, and (2) that their indications are liable to be disturbed by the heating effect of the current employed. These objections undoubtedly exist, and have frequently proved fatal to the employment of resistance thermometers : but they have arisen chiefly from faulty application or construction, and not from defects inherent in the method. Objection (1) is readily surmounted by proper methods of construction, and objection (2) by a proper consideration of the conditions of sensitiveness.

\section{Conditions of Sensitiveness.}

2. The conditions of sensitiveness in measuring a resistance by the Wheatstone bridge method have been discussed by Maxwell ('Electricity and Magnetism,' vol. i. p. 437) and Heaviside (Phil. Mag. Feb. 1873), whose results have generally been quoted and applied to the problem under consideration. They start with the assumption that the battery power available is linited by the internal resistance of the cells, and give rules for obtaining the maximum current through the galvanometer with this implied limitation. The limitation of battery power was no doubt an important consideration in many kinds of telegraph testing thirty years ago, but it is rarely applicable in modern laboratory practice, and never in dealing with electrical resistance thermometers. In the majority of resistance measurements, and more particularly in electrical thermometry, the limiting condition is imposed by the heating effect of the current on the resistance to be measured, and the resistances, etc., should be chosen and arranged to give the greatest 
sensitiveness for a given limiting value of the current through the resistance to be measured. The resistance of the battery circuit is quite immaterial provided that the battery can be arranged to give the required limiting current. The problem has been discussed from this point of view by Guye (Arch. Sci. Phys. Nat. Geneva, 1892), and by Schuster (Phil. Mag. xxxix. p. 175, 1893), and in several of my own papers, but it will facilitate discussion to reproduce here the investigation itself in a simplified form.

Let the annexed diagram, fig. 1, represent the arrangement of resistances in a Wheatstone bridge, in which $C^{\circ}$ is the

Fig. 1.

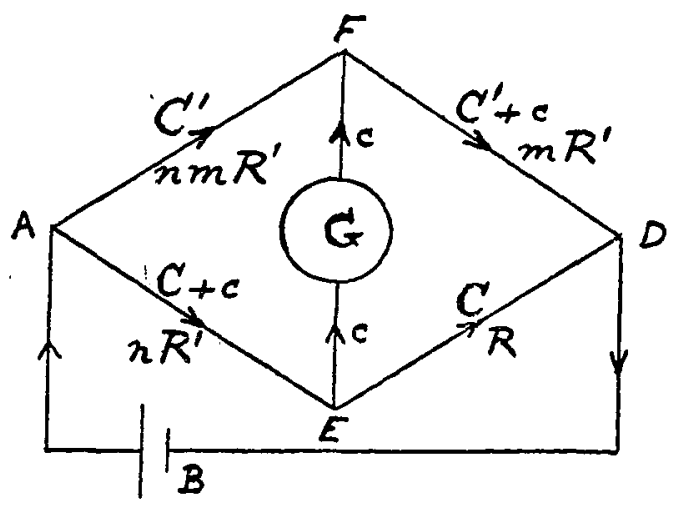

Diagram of Wheatstione Bridgs.

current through the resistance to be measured $R$, and $c$ is the current through the galvanometer of resistance $G$, when the bridge is not balanced. The resistance in series with $R$ is $n \mathrm{R}^{\prime}$ traversed by a current $\mathrm{C}+c$; the resistance in parallel with $\mathrm{R}$ on the same side of the galvanometer circuit is $m R^{\prime}$ traversed by a current $\mathrm{C}^{\prime}+c$. The resistance in the opposite arm of the bridge to $\mathrm{R}$ is $n m \mathrm{R}^{\prime}$, traversed by a current $\mathrm{C}^{\prime}$. The currents are assumed to flow in the directions indicated by the arrows, and it is evident that they satisfy the condition of continuity. The numbers $n$ and $m$, representing the ratios of the arms of the bridge, may have any positive values. The bridge is balanced when $R=R^{\prime}$, in which case $c=0$, and $\mathrm{C}^{\prime}=\mathrm{C} / \mathrm{m}$.

Since the difference of potential betwcen the ends of the 
galvanometer circuit is $G_{c}$, we have by Ohm's law,

$$
\mathrm{RC}-m \mathrm{R}^{\prime}\left(\mathrm{C}^{\prime}+c\right)=\mathrm{G}_{c}=n m \mathrm{R}^{\prime} \mathrm{C}^{\prime}-n \mathrm{R}^{\prime}(\mathrm{C}+c) .
$$

Eliminating $\mathrm{C}^{\prime}$, we obtain for the ratio $c / C$,

$$
c / \mathrm{C}=\left(\mathrm{R}-\mathrm{R}^{\prime}\right) /\left(\mathrm{G}(1+n) / n+(1+m) \mathrm{R}^{\prime}\right) . .
$$

It is obvious that this ratio, which may be regarded as a measure of the sensitiveness of the arrangement, is quite independent of the resistance or E.M.F. in the battery circuit. It is also at once evident that, for a given defect of balance as measured by $R-R^{\prime}$, the value of the ratio $c / C$ will be a maximum when $n$ is as large as possible, and $m$ as small as possible, the limiting value of the ratio $c / \mathrm{C}$ in this case being, when

$$
n=\infty, \text { and } m=0, c / \mathrm{C}=\left(\mathrm{R}-\mathrm{R}^{\prime}\right) /\left(\mathrm{G}+\mathrm{R}^{\prime}\right) .
$$

This point has been emphasized by both Guye and Schuster, and comes out clearly in Heaviside's investigation.

The advantage which can be gained by making $n$ large and $m$ small is not very great, because when $n=m=1$,

$$
c / \mathrm{C}=\left(\mathrm{R}-\mathrm{R}^{\prime}\right) / 2\left(\mathrm{G}+\mathrm{R}^{\prime}\right),
$$

or the sensitiveness is still half as great as in the limiting case.

For practical purposes it is more important to observe that if $n$ is smitl or $m$ large, the sensitiveness may be indefinitely reduced. This arrangement should always be avoided if possible, as shown by the following example.

\section{Maxwell's Rule.}

3. Maxwell has given a rule which is very often quoted and applied ('Electricity and Magnetism,' vol. i. sectiun 348 ). "Of the two resistances, that of the battery and that of the galvanometer, comnect the greater resistance so as to join the two greatest to the two least of the four other resistances."

This rule is seldom applicable to accurate resistance measurements, and when applied in platinum thermometry has often led to disastrous results. A simple numerical example will make this clearer.

Suppose that it is required to measure the resistance of a VoL. XXII. 
platinum thermometer, $R=10 \mathrm{ohms}$, with a post-office box having ratio arms 10 and 1000 ohms. Suppose that the galvanometer has a resistance $G=10 \mathrm{ohms}$, and that the battery is a 2 volt cell of negligible resistance. According to Maxwell's rule we should connect the galvanometer so as to join the two resistances of $1000 \mathrm{ohms}$ to the two resistances of $10 \mathrm{ohms}$, in which case we should have $n=1$, and $m=100$ in fig. 1. Suppose that the balance is slightly disturbed by a change $d R=R-R^{\prime}$. Substituting in equation (1) we obtain :

Case (A), Maxwell's Rule,

$$
n=1, m=100, c / \mathrm{C}=d \mathrm{R} / 1030, \mathrm{C}=0.1 \mathrm{amp} \text {. }
$$

If, however, we interchange the connexions of the battery and galvanometer, which is simply equivalent to interchanging the values of $n$ and $m$ in the equation, we obtain for the Anti-Maxwell arrangement:

Case (B), Anti-Maxwell,

$$
n=100, m=1, c / \mathrm{C}=d \mathrm{R} / 30, \mathrm{C}=\cdot 002 \mathrm{amp} .
$$

The actual value of the current through the galvanometer in case (A) is greater than in case (B) in the proportion of 3 to 2. Maxwell's rule is so far justified. But the current through the resistance to be measured is 50 times greater in (A) than in (B), and the heating effect of the current is 2500 times greater. With an orlinary platinum thermometer arrangement (A) might give an elevation of temperature of about $5^{\circ} \mathrm{C}$., which would be fatal for any purpose of measurement. Whereas arrangement (B), with nearly the same sensitiveness, would give an elevation of $\cdot 002^{\circ} \mathrm{C}$. only, which is sufficiently small for the most accurate work.

The essential point is to observe that the ratio $c / C$ of the galvanometer current to that throngh the thermometer, which is the true measure of sensitiveness in this case, is 34 times greater in (B) than in (A). (B) is therefore by far the better arrangement in the case where the heating effect of the current on the resistance to be measured is the primary consideration.

The rule to replace Maxwell's in this case, if there is any choice as to the arrangement of the battery and galvanometer connexions, is: "Connect the battery so as to make the 
resistance in series with the thermometer greater than the resistance in parallel." This will make $n$ greater than $m$ in fig. 1 , and it is evident that the ratio $c / C$ will be diminished or the sensitiveness reduced, if $n$ and $m$ are interchanged.

In the practical use of platinum thermometers, we have the further restriction that $n$ must be equal to unity, because it is necessary to compensate the changes of resistance of the leads by the equal changes of resistauce of a pair of compensating leads on the opposite side of the galvanometer contact on the bridge-wire. The loss of sensitiveness, as compared with the case of $n$ very large, does not amount to more than 20 or 30 per cent., and is of no consequence as compared with the trouble and uncertainty involved in measuring the resistance of the leads separately at each observation. Moreover, if $n$ is made large, the heating effect of the current $\mathrm{C}$ on the resistance $n \mathrm{R}^{\prime}$ may become serious. As a matter of fact this is a common and very insidious source of error in the use of a P.O. box with $n=1.00$, especially if the coils are of german silver or platinoid. With maganin resistances the effect is much less market.

It is still possible to gain some advantage in point of sensitiveness by making $m$ smaller than unity, provided that it is not made so small that the heating effect of the current $\mathrm{C} / m$ on the resistance $m \mathrm{R}^{\prime}$ becomes appreciable. For the most accurate work I have generally employed thermometers having a resistance about 25.6 ohms at $0^{\circ} \mathrm{C}$., with a fundamental interval of $10 \mathrm{ohms}$, and have made the ratio coils about $6.4 \mathrm{ohms}$, giving $m=1 / 4^{*}$, which gives an advantage

* The reason for choosing this particular value of $m$ in the case of the compensated box with platinum-silver aud platinum coils, which was exhibited at the Royal Society in May, 1893, and fully described later (Phil. Trans. R. S. A., rol. 199, p. 92), was that the change of resistance of the platinum-silver coils due to the heating effect of the current might be of the same order of magnitude as that of the platinum coils, and might take effect at the same rate. The thermal capacities and radiating surfaces of the coils being nearly equal, but the temperature coefficient of the platinum-silver nearly 16 times less than that of the platinum, the platinum-silver coils would carry about 4 times as great a current as the platinum for the same change of resistance. This was found to give a fairly safe limit for the value of $m$, but the effect was so small as to be of little importance. 
of aboat 20 per cent. in point of sensitiveness as compared with $n:=1$. A more important consideration in this case is that the same box can be used with thermometers of lower resistance, e.g. $R_{0}=2.56 \mathrm{ohms}, F . I .=1 \mathrm{ohm}$, without serious loss of sensitiveness.

\section{Resistance of Galvanometer.}

4. It is generally possible to vary the resistance of a galvanometer through a wide range, by altering the connexions of the coils or by substituting one coil for another, without altering the mass of the coils. In this case the sensitiveness will vary approximately as the square root of the resistance $G$. We see from equation (1) that the deflexion of the galvanometer, which is proportional to $c \sqrt{\mathrm{G}}$, will be a maximum when

$$
\mathrm{G}=n(1+m) \mathrm{R}^{\prime} /(1+n) .
$$

This result agrees with that given by Maxwell, and shows that in the majority of cases which occur in practice, $G$ should be of the same order of magnitude as $R$, being restricted to the limits $2 \mathrm{R}$ and $\mathrm{R} / 2$, if $n$ is never less than 1 , or $m$ greater than 1 . If we are restricted, as in platinum thermometry, to the case $n=1$, we should take

$$
\mathrm{G}=(1+m) \mathrm{R}^{\prime} / 2 .
$$

The best resistance for the galvanometer is restricted to the same limits, $2 \mathrm{R}$ and $\mathrm{R} / 2$, provided that $m$ does not exceed 3 .

For example, in the case of the box already quoted, with ratio coils 6.4 ohms, when used with a thermometer $R_{0}=25.6$ ohms, the best resistance for the galvanometer was $16 \mathrm{ohms}$. When the same box was used with a thermometer ten times smaller, $R_{0}=2.56$ ohms, the best resistance for the galyanometer would be 4.5 ohms. This could be secured approximately by putting the two coils of the $16 \mathrm{ohm}$ galvanometer in parallel, but the advantage gained thereby would be only 10 per cent. If, on the other hand, the ratio coils had been made equal to $25.6 \mathrm{ohms}$ each, according to the rule given by Maxwell and generally followed, there would have been a loss of sensitiveness of 20 per cent. with the $25.6 \mathrm{ohm}$ thermometers, and 50 per cent. with the $2.56 \mathrm{ohm}$ thermometers, which could not so well be neglected. 
Resistance of the Thermometer.

5. For a platinum thermometer of resistance $R_{0}$ at $0^{\circ} \mathrm{C}$, the change of resistance per $1^{\circ} \mathrm{C}$. is approximately $\left.{ }^{\circ} 01\right) 4 \mathrm{R}_{0}$, which must be substituted in equation (1) for the value of $d \mathrm{R}$ or $\mathrm{R}-\mathrm{R}^{\prime}$, in order to find the deflexion of the galvanometer per degree change of temperature. Making this substitution, and remembering that $n=1$, we find that, when the resistance of the thermometer is changed, the deflexion per degree, which is proportional to $c \sqrt{G}$, varies as $\mathrm{RC} \sqrt{\mathrm{G}} /(2 \mathrm{G}+(1+m) \mathrm{R})$.

The rise of temperature produced in the thermometer by the measuring current $C$ is directly proportional to $C^{2} R$, and inversely proportional to the rate of dissipation of heat per degree rise of temperature of the thermometer above its surroundings. The rate of dissipation of heat depends on the form and surface of the thermometer, and on the conditions of exposure. For similar thermometers of different resistances, but of the same size, under similar conditions of exposure, we must have $C^{2} R$ the same in order to secure the same degree of accuracy as limited by the heating effect of the current. The permissible current $\mathrm{C}$ will therefore vary inversely as the square root of the resistance of the thermometer. Making this substitution, we have

$$
c \sqrt{G} \text { varies as } \sqrt{\mathrm{GR}} /(2 \mathrm{G}+(1+m) \mathrm{R}) \text {. }
$$

We see from this result that, if it is possible to choose the best resistance for the galvanometer, namely $(1+m) \mathrm{R} / 2$, and if $m$ is the same for all, the sensitiveness and accuracy of the thermometer, so far as the heating effect of the current is concerned, will be independent of its resistance. In accurate laboratory work, where a delicate galvanometer is available, the heating effect of the current is so small as to be relatively unimportant, and the resistance of the thermometer is chosen chiefly with a view to minimise errors due to defects of insulation or imperfect contacts, or small differences in the resistance of the thermometer and compensator leads. On the other hand, for practical applications, or for ink recording, it is necessary to employ a fairly robust and portable galvanometer of the suspended or pivotted coil type ; the current employed must be considerably increased 
if' a large scale record is required, and it may be importan to choose the resistance of the thermometer to suit the galvanometer or vice versa, in order to minimise the heating effect of the current.

The symmetry of the above expression in respect of $G$ and $R$ shows that the condition governing the choice of the resistance of the thermometer, if the galvanometer is given and $m$ is constant, is the same as that already given for the galvanometer in terms of $R$, namely,

$$
\mathrm{R}=2 \mathrm{G} /(1+m) \text {. }
$$

If, however, the value of $m \mathrm{R}=\mathrm{S}$ is constant, being fixed by the resistance of the ratio coils, each equal to $S$, of the bridge employed, the maximum sensitiveness is obtained when

$$
R=2 G+S .
$$

The latter condition gives the linit beyond which no advantage can be gained by increasing $R$ and diminishing $m$, even if the heating effect of the current on $S$ can be entirely neglected. If the heating effect of the current in the thermometer is kept constant, as already assumed, the heating effect in $\mathrm{S}$ increases in proportion to $\mathrm{R} / \mathrm{S}$. On the same assumption the total current to be supplied by the battery (which is a consideration in records of long duration) is a minimum when $R=S$, but is only increased in the ratio $5 / 4$ when $R=4 \mathrm{~S}$. The maximum sensitiveness is obtained when $S=0$ and $R=2 G$, but the sensitiveness will be reduced hy little more than 10 per cent. if we make $S=G / 2$, keeping $\mathrm{R}=2 \mathrm{G}$. It would be reduced a further 20 per cent. if we made $\mathrm{R}=\mathrm{G}=\mathrm{S}$, according to Maxwell's investigation *.

In employing the deflexion method described below, the advantage of making $R$ larger and $S$ smaller than $G$ is really greater than would appear at first sight, because, is the sensitiveness is reduced 20 per cent. for the same heating effect of the current, it means in practice that the current must be increased 20 per cent. to give the required deflexion, or that the heating effect of the current must be increased

- Maxwell, 'Electricity and Magnetism,' vol. i. §349. This is the right arrangement if all the four arms of the bridge are similar, and equally affected by the current. 
more than 40 per cent. The most important error to avoid is to make $R$ small compared with $G$ or $S$. The sensitiveness may in this way be very greatly reduced, and the heating effect of the current increased five or ten times. Thus, a pyrometer of low resistance designed for work at high temperatures (where the resistance is high and an open scale is not required) would not be a suitable instrument to employ for recording small variations of temperature on an open scale at low temperatures, unless $G$ and $S$ were made inconveniently small, and the pyrometer itself specially wound with extra thick wire to give a large cooling surface.

\section{Construction of 7 hermometers.}

6. The construction of the thermometers depends on the situation in which they are to be used. I have made three principal types:-(1) For insertion in the mouth; (2) for the rectum ; (3) for the surface of the skin or for the axilla (armpit).

(1) The mouth is not a suitable position for records of long duration, but it is of interest to be able to insert an electrical thermometer in this way in order to investigate the conditions of lag, which cannot be observed so accurately with a mercury clinical thermometer. It is most essential that the thermometer should be of small thermal capacity, in order to minimise the effect of inserting a cold thermometer, since the tissues of the body are not very good conductors of heat. In any case, if the mouth has not been kept shut for some time previous to the insertion of the thermometer, there will be a large apparent lag due to the recovery of temperature of the mouth itself. A so.called half-minute clinical thermometer may take upwards of five minutes to get within a half a degree of the true temperature under these conditions. I have tested several recording thermometors from this point of view, and the results are of some interest.

Flat Glass Bulb Thermometer.-The ordinary type of platinum thermometer has the wire wound on a mica cross with eompensated leads insulated by mica disks. Thermometer and leads are slipped into a containing tube about $10 \mathrm{~mm}$. diam., which is removable, and can easily be replaced 
if damaged or broken, and which permits withdrawal of the thermometer for adjustment or repair, if required. This type is most convenient and suitable for ordinary laboratory work with sensitive galvanometers; but is insufficiently sensitive for insertion in the mouth. Moreover, since the wire is surrounded by air, the heating effect of the current may be excessive when such a thermometer is used with recording instruments on an open scale, especially if its resistance is low.

A great improvement on this type, in point of quickness of action and diminution of the heating effect of the current, is readily effected by winding the wire on a flat plate of mica in place of a cross, and melting the lower part of the containing tube (which is preferably of lead glass) down on to the wire so as to form a flat bulb, as illustrated in fig. 2. If the glass is thin, the sensitiveness may be increased

Fig. 2.

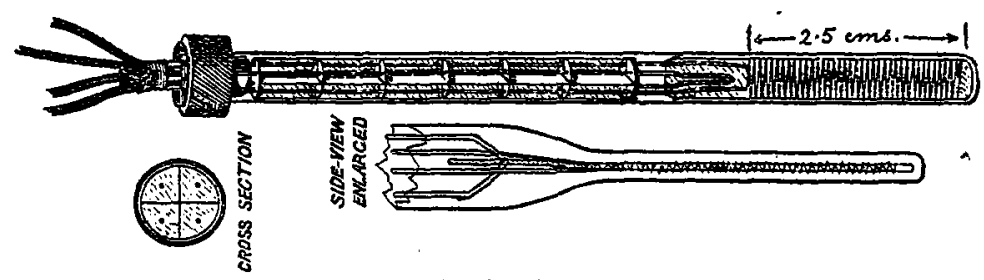

Flat Glass Bulb Thermometer.

nearly five times, as compared with a thermometer of the ordinary type, and the heating effect of the current reduced in nearly the same proportion. When inserted in a waterbath at $37^{\circ} \mathrm{C}$. this thermometer takes less than a minute in arriving within a hundredth of a degree of the final temperature; but when inserted cold in the mouth, it may take 4 or 5 minutes to get within a tenth of a degree, because the tissues of the mouth take time to recover from the cooling effect of inserting the thermometer, as further illustrated below. Where quickness of action is an essential condition, as in some kinds of calorimetric work, the flatbulb thermometer is a great improvement on the ordinary type with a round tube, but it shares with the mercury thermometer the disadvantage that, if broken or damaged, it cannot easily be repaired. 
It is essential that thermometurs of this type should be provided with compensated leads, otherwise there will be a variable immersion error, and an apparent lag due to slow conduction of heat along the leads. It is also most important for accurate work to avoid screw terminals in the head of the thermometer, and to provide each instrument with flexible leads two or three metres long, permanently soldered to the thermometer and compensator leads, and securely attached to the head of the thermometer, as indicated in fig. 2.

Herceus Quartz-Glass Thernometer.-Whe smallest size of quartz-glass resistance thermometer constructed by Heræus after the experiments of Dr. Haagn has a bulb $25 \mathrm{~mm}$. long and $3 \mathrm{~mm}$. in diameter, and is therefore very similar in dimensions to an ordinary clinical thermometer. The bulb contains a coil of fine platinum wire wound on a rod of quartz-glass, and protected by a very thin tube of quartzglass fused over it. The bulb is fused on to a larger quartz tube, containing the two leading wires, which are of gold. The resistance of the coil is $25 \mathrm{ohms}$ nearly at $0^{\circ} \mathrm{C}$., but, as there are no compensating leads, the resistance cannot be measured very accurately. This type of thermometer is very suitable for insertion in the mouth, but is unsuitable for records of long duration owing to its shape and the risk of breakage. It does not appear to be quite so sensitive as a mercury thermometer of similar dimensions, partly owing to the low conductivity of the central rod of quartz-glass, and partly to the fact that the leads are not compensated. When inserted in a water-bath at $37^{\circ} \mathrm{C}$. this thermometer starts very quickly to rise, but takes about a minute in arriving within a tenth of a degree C. of its final reading, and continues to change appreciably for three minutes, possibly owing to slow conduction along the leads, one of which passes through the centre of the coil. When placed under the tongue, after keeping the mouth closed for ten minutes previously, it arrives within a degree of the final temperature in one minute, but takes seven or eight minutes to get within a tenth of a degree. A typical record obtained with this thermometer is given in fig. 3.

Flat Platinum Tube Thermometer.-This is a type of thermometer which I specially designed for calorimetric 
work, where quickness of action and small mass are important. It is similar to the flat glass bulb thermometer, except that the containing tube is of platinum about $0.05 \mathrm{~mm}$. thick, which permits the thermal capacity of the bulb to be greatly reduced. The coil is wound on a flat plate of very thin mica, and insulated from the flattened platinum tube by thin strips of mica on either side. The lag of this thermometer when placed in a water-bath at $37^{\circ} \mathrm{C}$. was so much less than that of the galvanometer that it could not be recorded satisfactorily. Owing to its size and shape, it was not very suitable for insertion in the mouth, as it could not be placed in the usual position under the tongue. When placed along the side of the mouth (after closure for ten minutes) between the tongue and the teeth, it arrived within $0^{\circ} .3$ of the final temperature in less than a minute, but continued to rise appreciably for nearly ten minutes, apparently indicating that the mouth had not been kept closed long enough beforehand to reach a practically steady temperature.

A comparison of the records of the platinum tube thermometer (Pt) and the Heræus quartz tube thermometer $(\mathrm{He})$ when placed in the mouth under similar conditions is showr

Fig. 3.

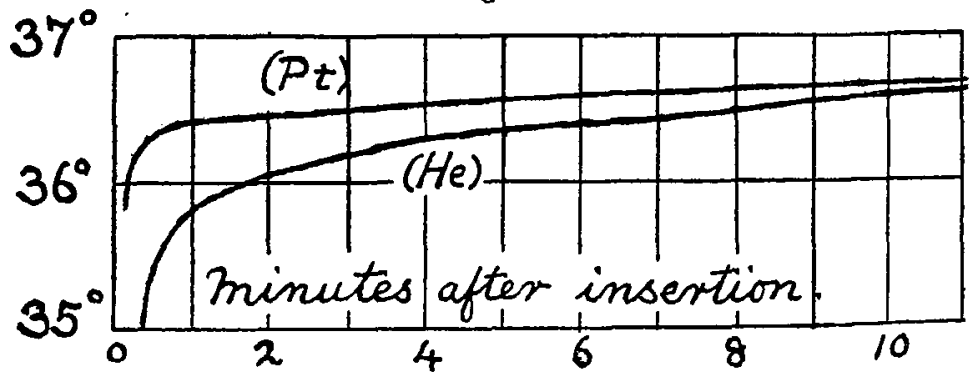

Comparison of Quartz and Platinum Tube Thermometers in Mouth after closure for 10 minutes.

in fig. 3, which illustrates the importance of extreme quickness and small thermal capacity in such tests.

It might appear at first sight as though the slow and long continued rise indicated by both thermometers in different 
degrees, were due to the heating effect of the measuring current, which might easily produce a result of this nature if the current were excessive or the mass considerable. But the current employed in taking these records scarcely exceeded one hundredth of an ampere in the case of either thermometer, and the heating effect of the current was measured in both cases and found to be very small. With the Heræus thermometer, when placed in a water-bath and traversed by the same current, the rise of temperature due to the passage of the current was found to be only $0^{\circ} .085 \mathrm{C}$. and to reach a steady value in about two minutes. When enclosed in its metal protecting tube, so that the bulb of the thermometer itself was surrounded by air instead of being in direct contact with the water, the heating effect of the current was increased to $0^{\circ} .31 \mathrm{C}$., and became steady in less than three minutes. The comparatively high value of the heating effect of the current with this thermometer is evidently due to its small size and surface. When placed in the mouth the heating effect of the current could not be determined accurately, as the temperature was not perfectly steady, but it was probably less than a tenth of a degree, and therefore insufficient to account for the observed lag. With the flat platinum tube thermometer, owing to its much larger surface, the heating effect of the current was far too small to be appreciable on the scale of the records.

That the apparent lag of the thermometer is really due to the slowness of recovery of the temperature of the mouth itself after being cooled in any manner, is furtber shown by the following records taken with the platinum tube thermometer under different conditions. The first curve, marked (1) in fig. 4 was taken at the conclusion of an ordinary conversation, without any precautions as to keeping the mouth closed or refraining from speaking, until the thermometer had been inserted. The thermometer did not reach $32^{\circ} \mathrm{C}$. for more than a ninute, and was still nearly $1^{\circ} \mathrm{C}$. below the normal temperature after a lapse of seven minutes. Curve No. (2) represents the record ohtained after keeping the mouth closed for three minutes previous to the insertion of the thermometer. Curve No. (3), which is practically a reproduction of the record already given in fig. 3 , represents 
the result of keeping the mouth closed for ten minutes previously. There is a further improvement with this thermometer after closure for fifteen minutes, but unless the mass of the thermometer is very small, or the thermometer itself is previously warmed, little advantage is gained by

Fig. 4.

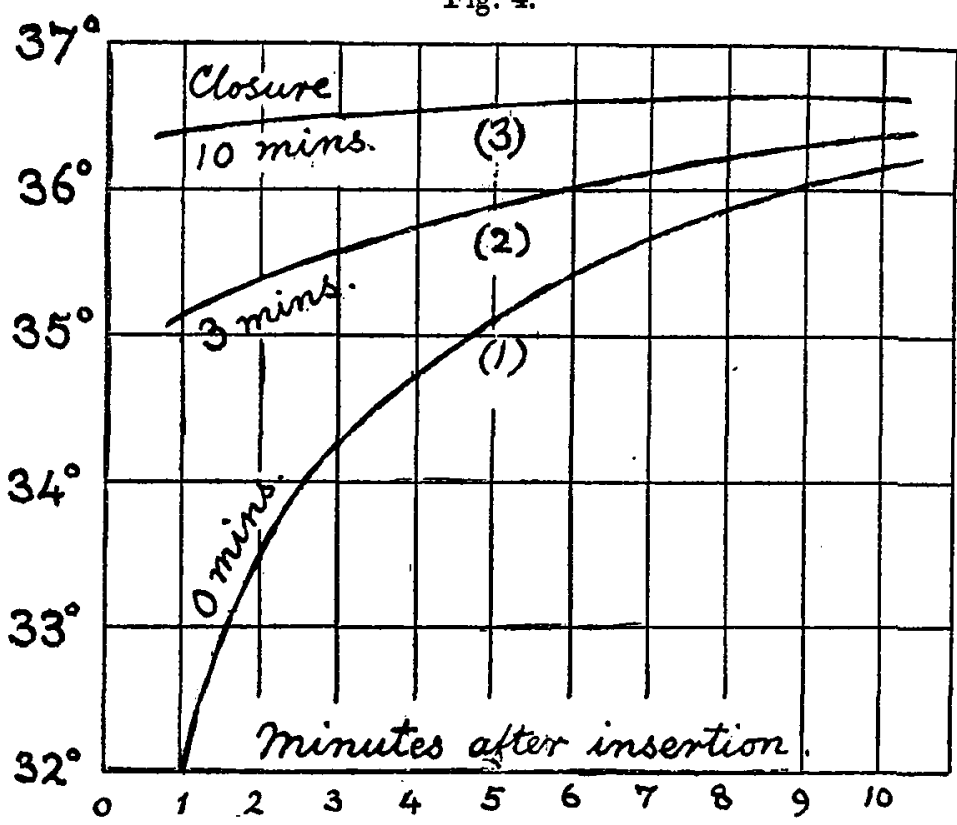

Platinum Tube Thermometer Records in Mouth after different periods of closure.

keeping the mouth closed more than ten minutes, because in any case the mouth takes some time to recover from the cooling effect of inserting the thermometer. In all the records given in figs. 3 and 4 , the thermometer was inserted cold, being taken direct from a water-bath at $15^{\circ} \mathrm{C}$. The difference between the records in fig. 4 is due almost entirely to an actual difference in the temperature of the mouth at starting. The difference between the quartz and platinum tube thermometers in fig. 3 is due chiefly to the greater cooling effect of the quartz tube, owing to its greater mass in proportion to its surface. 
(2) For records of long duration, the best results are undoubtedly to be obtained by insertion in the rectum. The thermometer bulb should be of small dimensions and the leading wires should be enclosed in a flexible but inextensible tube of small diameter, such that it can be attached to the bulb in a perfectly secure and water-tight manner. Such a thermometer may be made in a variety of ways. The following is a description of a method of construction which I have found very satisfactory. The fine wire forming the bulb is wound on a thin celluloid tube $5 \mathrm{~mm}$. in diameter, and is protected by another thin celluloid tube which closely fits the first. One end of the protecting tube is closed by a small celluloid stopper, the other is cemented with celluloid cement to the cut end of a Porges catheter $5 \mathrm{~mm}$. in external diameter, and $32 \mathrm{~cm}$. long, through which the flexible thermometer and compensator leads are passed. This type of catbeter has a smooth surface, and is very flexible, but practically inextensible, thus protecting the leading wires and joints from possible strain. It also makes a very good joint with the celluloid tube protecting the bulb. I have tried rubber tubes and metal bulbs, but these appear to be in every respect less suitable. A rubber stopper fixed on the catheter at a distance of 3 to $5 \mathrm{~cm}$. from the near end of the bulb, is a convenient method of limiting the depth of insertion. The thermometer is smeared with vaseline before insertion. This prevents irritation, and does not appear to have any injurious effect on the celluloid or catheter.

The curves shown in figs. 5 and 6 are typical records taken with this thermometer on consecutive nights under similar conditions. In the first case there was an unusually rapid rise in the external atmospheric temperature during the night which occasioned some feeling of discomfort. In the second case there was a sharp frost, and the patient awoke feeling appreciably chilled. From a comparison of similar records it would appear that the normal temperature of the body may be influenced to some extent, as one would naturally expect, by the external conditions prevailing at the time. There is often a fall of nearly half a degree $C$. during the night corresponding more or less with the diurnal change of atmospheric temperature. 
The changes here observed cannot have been due, as might appear at first sight, to the effect of change of temperature on the resistance coils of the box or measuring apparatus, because increase of resistance of the balancing coils in the

Fig. 5.

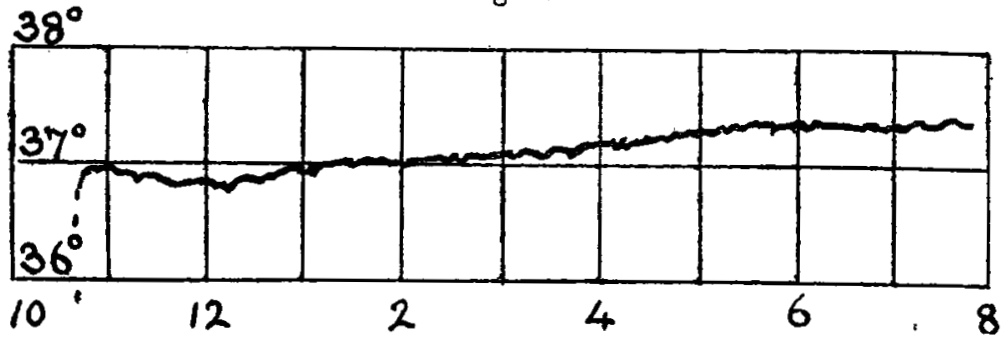

Rectal Thermometer on Thread Recorder.

Fig. 6.

$38^{\circ}$

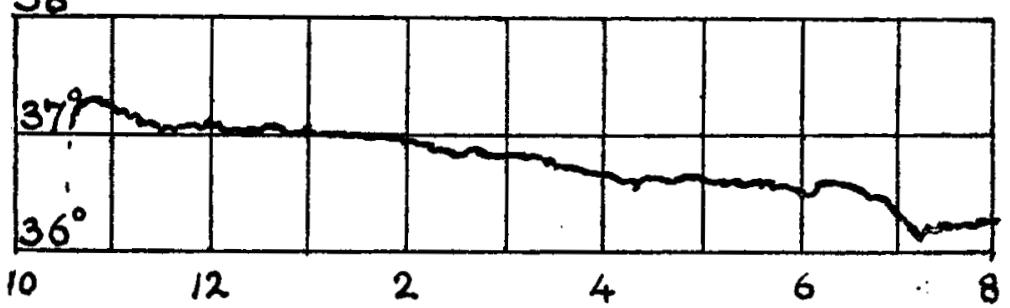

Rectal Thermometer on Thread Recorder.

bridge would act in the opposite direction, producing an apparent fall of the thermometer. Moreover, the balancing coil in this particular thermometer was made of fine manganin wire having a resistance equal to that of the thermometer coil at $37^{\circ} \mathrm{C}$, and was placed inside the celluloid tube on which the thermometer coil itself was wound, so that any changes in its resistance were due to change of temperature of the thermometer, and were allowed for when the thermometer was calibrated. This construction was adopted in order to avoid the necessity of applying any correction for change of temperature of the resistance box; but it is open to the objection that it makes the thermometer rather less sensitive, and doubles the heating effect of the current. The 
sensitiveness of the celluloid rectal thermometer, though only about half as great as that of the Heræus thermometer, was ample for records of long duration. The heating effect of the current was only a tenth of a degree, and did not introduce any appreciable error, since it remained practically constant, and was allowed for in the calibration, in which the same current was employed.

A more important source of systematic error would lie in variation of E.M F. of the battery during a long continued run. But unless the storage cells employed were nearly run dowa, this would not be likely to amount to more than 1 per cent. of the galvanometer deflexion, or two or three hundredths of a degree, since the galvanometer deflexion corresponds only to the small difference from the balance point which is in the neighbourhood of $37^{\circ} \mathrm{C}$. The zero of the galvanometer and the deflexion per degree would of course be tested, and, if necessary, adjusted daily.

(3) Surface or Axillary Type.-A tube form of thermometer is not very suitable for this purpose, since the contact between the thermometer and the skin is necessarily imperfect. It is desirable to give the thermometer as large a surface as possible, in order to diminish the heating effect of the current, and at the same time to make the bulb flexible and thin, so as to conform approximately to the contour of the surface to which it is applied. The first thermometer of this type which I constructed for Prof. Adami of Montreal in 1897 , was made by sticking a thin bolometer grid of platinum foil on to a thin sheet of celluloid photographic film. This satisfied the required conditions very perfectly, but the bolometer grid was excessively fragile (unless its resistance were made unduly low), and was also difficult to attach securely to the leading wires. The substitution of fine wire for the foil greatly facilitated the construction and adjustment, and was found to make little, if any, difference to the sensitiveness. In any case it is most important that the thermometer should be fairly robust, as it has to undergo somewhat rough usage. In my experience nothing answers so well as celluloid film for the insulating material. The wire may be protected by cementing over it a thin film of celluloid; but a better plan is to insert the thermometer in 
a thin flat celluloid sheath, as illustrated in fig. 7, which can be changed at any time if it becomes soiled. The flexible leads are flattened and insulated between celluloid films at the end where they join the thermometer coil. In applying the thermometer to the surface of the body, a truss, similar to that employed by Dr. Gamgee (loc. cit.) for thermocouples, may be used; but, in my experience, a simpler and more generally applicable method is to back the thermometer with a pad of cotton-wool to secure good contact, and to keep it in place by means of an elastic band to which the thermometer and leads are pinned or sewn. When the thermometer is placed in the left axilla, which is generally

Fig. 7.

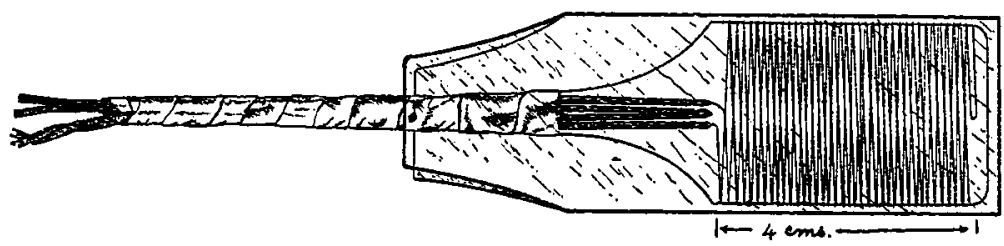

Axilla or Surface Thermometer (Celluloid).

the most convenient location, the elastic band should pass under the left arm, and over the right shoulder near the neck.

A surface thermometer of this type is extremely sensitive, and shows no appreciable lag when placed in a water-bath. But when placed in the axilla, or elsewhere on the surface of the skin, it may take fifteen minutes or more to reach a steady temperature. The reason of this is partly that the skin is slightly chilled by exposure during the insertion of the thermometer, and partly that the application of the thermometer with its wool pad tends to raise the temperature of the skin locally by preventing evaporation. The temperature of the skin under normal conditions with free evaporation is generally lower than that of the body. If the patient is ulready in bed and well covered up, the initial lag may be greatly reduced, but is in any case of little or no consequence for records of long duration. 
A thermometer of this type should not be heated to $100^{\circ} \mathrm{C}$. or directly exposed to water at temperatures as high as $50^{\circ} \mathrm{C}$, because this procedure is liable to buckle the film. If the temperature coefficient of the wire is known, it can be calibrated with sufficient accuracy by a single observation in a water-bath at the ordinary room temperature with a reliable mercury thermometer. If it is to be tested in a water-bath at $40^{\circ}$ or $50^{\circ} \mathrm{C}$, it should be protected by a thin copper sheath. I have generally employed thermometers adjusted

Fig. 8.

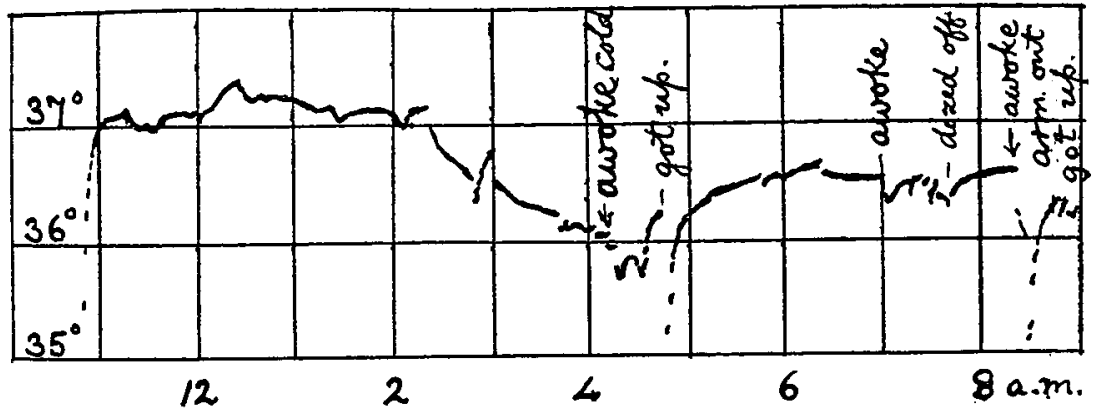

Axillary Thermometer A, with elastic band.

Fig. 9.

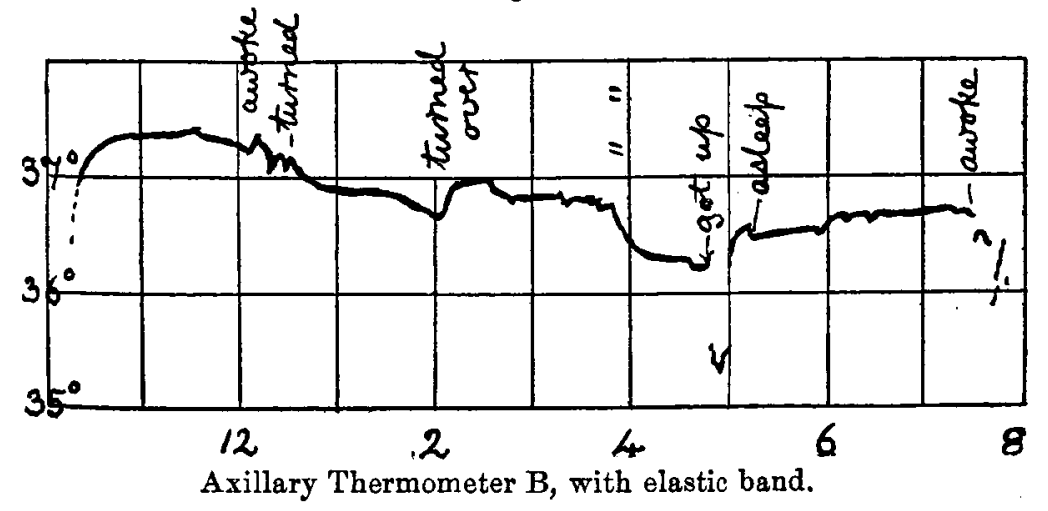

to a fundamental interval of $10 \mathrm{ohms}$, giving a change of one-tenth of an ohm per $1^{\circ} \mathrm{C}$. Each thermometer is provided with a balancing coil, the resistance of which is adjusted to be equal to that of the thermometer at or near $37^{\circ} \mathrm{C}$.

The chief difficulty in obtaining reliable records of the vOL. XXII. 
body temperature by means of a thermometer inserted in the axilla, is that the indications are liable to be disturbed by movements of the patient, more particularly by putting the left arm out of bed, or by raising the bed-clothes and admitting cold air in turning over from one side to the other. These effects are illustrated by the records shown in figs. 8 and 9, which were taken with different thermometers in the axilla on consecutive nights. The curve is often very smooth during sleep, but becomes irregular owing to greater restlessness on awaking. These accidental excursions seldom exceed a few tenths of a degree, unless the patient gets out of bed, and are chiefly due, in all probability, to slight displacements permitting intrusion of air betwen the thermometer and the skin. In the record sbown in fig. 10 a very

Fig. 10.

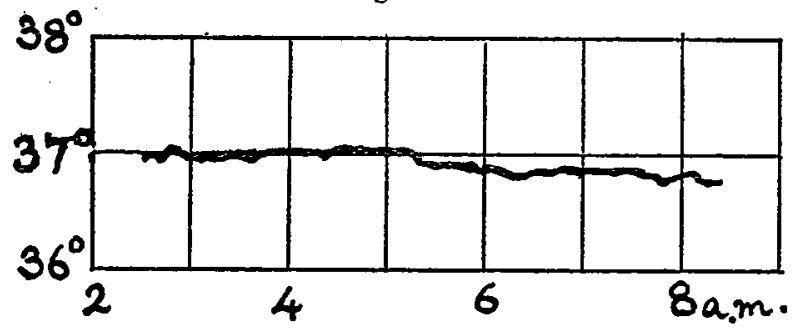

Axillary Thermometer $\mathrm{C}$, fastened in place with adhesive wax.

smooth curve was obtained by sticking the thermometer to the skin with adhesive wax. Unfortunately this record was of shorter duration, only six hours, and, as the patient did not get to bed until 2.30 A.M., it is possible that excessive fatigue may have induced unusual soundness of sleep.

\section{Recording Instruments.}

7. Two principal types of recording instruments are available, corresponding respectively to the Deflexion Method, and the Balance Method of measuring variations of electrical resistance. In the deflexion method, the greater part of the resistance to be measured is balanced on a Wheatstone bridge by a balancing coil, and the small variations are observed by the deflexion of the galvanometer. In the balance method, 
the galvanometer contact is moved along a bridge-wire until balance is obtained. This method is not so quick for small variations, but is generally most suitable for work with resistance thermometers, and has the advantage that the scale is uniform and independent of the E.M.F. of the battery. In clinical thermometry, the range of temperature to be covered is so small that the deflexion method is generally applicable and the scale practically uniform. The scale is readily adjusted to read in degrees of temperature by means of a suitable rheostat in the battery circuit. If storage cells of sufficient capacity are employed there is little risk of error from variation of the E.M.F. of the battery. Thus, for clinical thermometry, there is little to choose between the balance and deflexion methods, if the instruments employed in either case are equally sensitive. In the installation of underground thermometers, buried at various depths in the soil at McGill College in 1894, the deflexion method was employed, as being the quickest, for the daily readings of each thermometer ; but the balance method was adopted, as being the most accurate, for taking continuous records of the diurnal variations when occasion required. In order to obtain a strictly continuous record by the deflexion method it is necessary to employ photography, but a practically continuous record may be obtained, as in the Thread Recorder made by the Instrument Co., Cambridge, by depressing the pointer attached to the galvanometer coil so as to mark its position on the record sheet by means of an inked thread at regular intervals of a minute, the index being free for the greater part of the time. For records of long duration the separate dots coalesce into a continuous curve if the variations are not too rapid.

\section{Adjustment and Use of the Thread Recorder.}

8. The standard type of Thread Recorder is a fairly robust instrument, having a resistance of about 10-12 ohms, and a scale of $80 \mathrm{~mm}$. to one millivolt. With a Copper-Constantan thermocouple, this instrument gives a scale of about $3 \mathrm{~mm}$. to $1^{\circ} \mathrm{C}$., which is rather too small for clinical records. With a resistance thermometer, it is easy to get a scale of $50 \mathrm{~mm}$. 
to $1^{\circ} \mathrm{C}$. if desired, but a scale of 10 to $20 \mathrm{~mm}$. is usually sufficient.

In using the thread recorder with a resistance thermometer, some form of platinum thermometer bridge with a slide-wire is required, and a rheostat or resistance box capable of fine adjustment between 30 and 50 ohms. This rheostat is connected in series with a 2 volt storage cell to the battery terminals (marked B on the bridge), and serves to adjust the scale of the record. The thread recorder is connected to the terminals marked $G$ on the bridge, one of which is connected between the equal ratio coils of $10 \mathrm{ohms}$ each, and

Fig. 11.

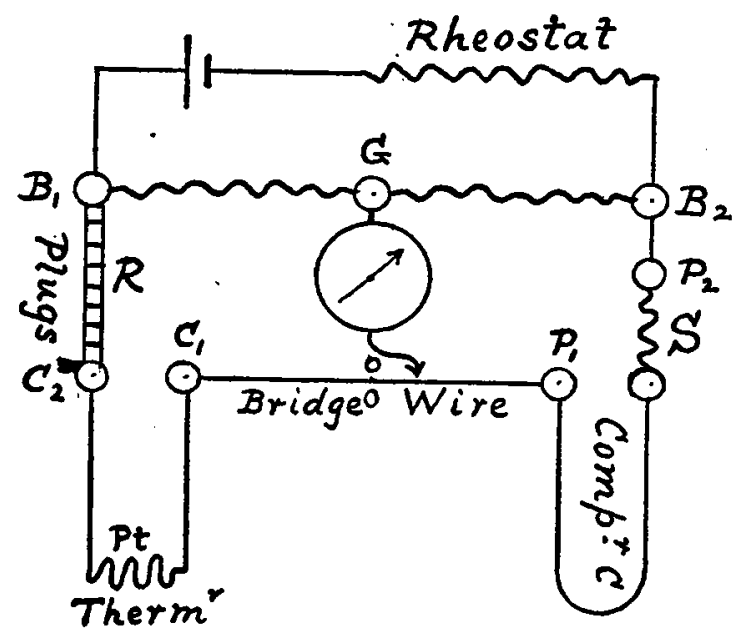

Diagram of Connexions for Thread Recorder.

the other to the sliding contact on the bridge-wire, as shown on the diagram, fig. 11.

The two thermometer leads are not connected to the terminals marked $\mathrm{P}$ on the bridge (which is the usual arrangement when the balance method is used), but to the terminals marked $\mathrm{C}_{1} \mathrm{C}_{2}$ (for compensator) so as to be in series with the adjustable plug resistances denoted by $R$ in the annexed diagram of connexions. The compensator leads are connected in series with the Balancing Coil, denoted by $\mathbf{S}$ in the diagram, to the terminals marked $P_{1} P_{2}$. With this 
arrangement the resistance $\mathbf{S}+\mathrm{C}$ is nearly constant, so that if the galvanometer deflexion is adjusted to be correct when the thermometer is at $0^{\circ} \mathrm{C}$. or $20^{\circ} \mathrm{C}$, or any convenient temperature, the scale will be very nearly correct when the thermometer is at any other temperature, the plugs $R$ being suitably adjusted.

As an example of the adjustment of the galvanometer scale, we will suppose that the thread recorder is properly set up and levelled, and the zero adjusted to the right hand side of the scale, which reads from right to left. The standard type of record sheet is divided into 25 scale-divisions, with a heavy line at each fifth division. With this sheet it is most convenient to adjust the scale to read 5 divisions to $1^{\circ} \mathrm{C}$., giving a range of $5^{\circ} \mathrm{C}$. on the record sheet. Suppose that the thermometer has a resistance of $26 \mathrm{ohms}$ at $0^{\circ} \mathrm{C}$., and is adjusted to have a fundamental interval of exactly $10 \mathrm{ohms}$, giving a change of $0.1 \mathrm{ohm}$ per $1^{\circ} \mathrm{C}$. Suppose that the balancing resistance $S$ is $30 \mathrm{ohms}$ and is adjusted to be equal to the thermometer at $40^{\circ} \mathrm{C}$. Ten centimetres of the bridge-wire, or ten units of the plug resistances $R$, will correspond to a tenth of an $\mathrm{ohm}$, or $1^{\circ} \mathrm{C}$, , on the thermometer.

To adjust the galvanometer scale, the thermometer may be placed in a vessel of water at the temperature of the room, and the bridge balanced. If the temperature is $15^{\circ} \mathrm{C}$. (which is $25^{\circ} \mathrm{C}$. below $40^{\circ} \mathrm{C}$.) it will be necessary to unplug 250 units (equivalent to $25^{\circ}$ ) in the box at $\mathrm{R}$ to balance $\mathrm{S}$, when the sliding contact is at zero in the centre of the bri lgewire. If balance were obtained with 230 units in $R$, and the sliding contact at $7 \cdot 3 \mathrm{~cm}$. to the right of zero, it would mean that the temperature of the water was $40-23+\cdot 73$ $=17^{\circ} .73 \mathrm{C}$. In any case, to obtain a fine adjustment for the balance, the sliding contact must be shifted till the galvanometer shows no deflexion, and then clamped in position. The galvanometer being thus balanced at a steady temperature, increase the resistance unplugged in $\mathrm{R}$ by 50 units $(0.5 \mathrm{ohm})$, corresponding to a rise of temperature of $5^{\circ} \mathrm{C}$. on the thermometer. If the adjustment of the rheostat is correct the galvanometer should deflect $5^{\circ}$ to the left, i.e. to the extreme left of the record sheet. If the galvanometer 
deflects to the right the connexions of its terminals must be reversed. If the deflexion is less than $5^{\circ}$, the resistance of the sliding rheostat must be reduced, and vice versa, until the desired deflexion is obtained. The adjustment will then be correct for any temperature within the range of the instrument up to $40^{\circ} \mathrm{C}$. To get a range on the record sheet from $35^{\circ}$ to $40^{\circ} \mathrm{C}$., for records of normal body temperature, it would then be necessary to unplug 50 units in $R$ and to set the bridge-wire contact at zero, so that the zero of the galvanometer scale may correspond to $35^{\circ} \mathrm{C}$. Similarly for a range from $40^{\circ}$ to $45^{\circ} \mathrm{C}$., the resistance unplugged in $\mathrm{R}$ should be zero. Temperatures above $45^{\circ}$ could be measured by increasing the balancing resistance $\mathrm{S}$, but it is not advisable to go above $45^{\circ} \mathrm{C}$. with celluloid thermometers.

The accuracy of adjustment of the thermometer and its balancing coil $S$ can be tested at any time by immersing the thermometer either in melting ice, or in water at the room temperature if a reliable mercury thermometer is available. If, for instance, the galvanometer reads zero when the thermometer is in water at $17^{\circ} .67 \mathrm{C}$. with 230 units unplugged, and the contact at $73 \mathrm{~mm}$. (reading $17^{\circ} .73 \mathrm{C}$.), the thermometer reads $6 \mathrm{~mm}$. or $\cdot 06^{\circ} \mathrm{C}$. too high at this temperature, and the error may be corrected by setting the bridge-wire contact $6 \mathrm{~mm}$. to the right of zero for the record, instead of at zero. Several different thermometers may be used with the same balancing coil, provided that allowance is made in this way for the small differences between them.

\section{Testing for Defects.}

9. To test the thermometer and compensator leads for defective insulation disconnect one of the thermometer leads, say $\mathrm{C}_{2}$, from the battery, and the opposite compensator lead, say $\mathrm{P}_{1}$, from the bridge-wire (or vice versa, $\mathrm{C}_{1}$ from the bridge-wire and $\mathrm{P}_{2}$ from the battery). If, now, the galvanometer circuit is made, there should be no deflexion if the insulation is perfect, since the bridge-wire and galvanometer contact are effectively isolated from the rest of the circuit, unless there is defective insulation between the thermometer and compensator. Before disconuecting the wires for this test the galvanometer circuit should be broken, otherwise the 
instrument $w i l l$ be violently agitated, and its zero may be affected.

To measure the heating effect of the working current on the thermometer, place the thermometer in water at a steady temperature, balance the bridge as already explained, and adjust the galvanometer scale if this has not been done. With the galvanometer balanced at zero, and the current adjusted to its proper value, change the battery from one storage cell to two cells of the same E.M.F. Since the resistance of a storage cell is negligible compared with the other resistances in the circuit, this change will have the effect of doubling the current through the thermometer. The rise of temperature of the thermometer due to the passage of the current will be quadrupled, since it varies as the square of the current. The change of resistance of the thermometer due to changing from one cell to two, will be three times the change of resistance due to the heating effect of the current with one cell. The deflexion of the galvanometer for a given change of resistance will also be doubled, since the current is doubled. The deflexion of the galvanometer produced by changing trom one cell to two will therefore be six times the heating effect with one cell measured in degrees of temperature. Hence observe the galvanometer deflexion in degrees of temperature and divide by six to find the heating effect for the normal current with one cell. If the heating effect so deduced does not exceed a tenth of a degree, it will not introduce any appreciable error, since it remains practically constant, so long as the scale adjustment is the same. With a given thermometer and galvanometer the heating effect varies as the square of the number of scale divisions per degree. Thus if the heating effect were found to be a tenth of a degree with the galvanometer adjusted for a scale of $2 \mathrm{~cm}$. to $1^{\circ} \mathrm{C}$., it would. be four tenths if the scale were increased to $4 \mathrm{~cm}$. per degree, and the zero would be raised three tenths.

\section{Culibration of the Scale of the Galvanometer.}

The angular deflexion of the galvanometer for one millivolt is rather large, and the scale may not be accurately one of equal parts. This should be tested for accurate work. 
With the aid of the bridge it is easy to make the test, either by placing the thermometer in water at a steady temperature, or by substituting a fixed resistance, equal to the balancing coil, in place of the thermometer. Balance the galvanometer at zero by adjusting the bridge-wire contact. Adjust the scale to the required value. Unplug successively resistances in $\mathrm{R}$ corresponding to $1,2,3,4,5^{\circ}$, and observe the galvanometer reading for each on the record sheet. Repeat in descending order to test the galvanometer for change of zero due to imperfect elasticity of the suspension. The errors should not as a rule be appreciable on the scale of the record unless there is something wrong with the levelling or suspension. It is of course extremely important that the galvanometer coil should be perfectly free in all positions of the boom, and it must be remembered that a variation of level may cause a variation of scale, as well as of zero.

\section{Balance Methad with Slide-vire Recorder.}

10. This is the type of recorder generally employed with resistance thermometers, especially where a large range of temperature is to be corered. It is equally applicable for clinical work, or for small ranges of temperature, provided that a suitable thermometer is employed, and that the current is properly adjusted to avoid excessive heating of the thermometer. It gives a strictly continuous ink record, with a pen attached to the sliding contact on the bridge-wire, which is automatically maintained at the balance point by means of a pair of motor clocks actuated by the deflexion of the galvanometer. The galvanometer coil carries a light arm terminating in a contact fork consisting of two short platinum wires close together. A wheel with a platinum edge rotates between these two wires, one or other of which makes contact with the wheel (according to the direction of deflexion of the galvanometer), and completes a relay circuit actuating one or other of the motor clocks. This form of relay is incredibly delicate, and has since been adapted by Brown for the purpose of submarine telegraphy. The contacts keep themselves clean automatically, and never require adjustment. Since the deflexion of the galvanometor is limited to a fraction of a millimetre, there is no 
chance of accidental disturbance of the zero by excessive deflexion.

The scale of the instrument is independent of the E.M.F. of the battery, being determined by the resistance of the bridge-wire in relation to that of the thermometer. The bridge-wires are usually made with a scale of $2 \cdot 0,1 \cdot 0$, or $0.5 \mathrm{ohm}$ to a length of $20 \mathrm{~cm}$., which is the length of the record sheet. With a thermometer having a zero resistance of $26 \mathrm{ohms}$, and a fundamental interval of $10 \mathrm{ohms}$, these bridge-wires give ranges of $20^{\circ} \mathrm{C}$., $10^{\circ} \mathrm{C}$, and $5^{\circ} \mathrm{C}$., respectively on the record sheet, or scrles of 1,2 , and $4 \mathrm{~cm}$. to $1^{\circ} \mathrm{C}$. With thermometers of smaller resistances the scales are proportionately smaller and the ranges larger. For clinical work the most convenient range is from $35^{\circ}$ to $45^{\circ} \mathrm{C}$., with a scale of $2 \mathrm{~cm}$. to $1^{\circ} \mathrm{C}$. This is obtained with a $26 \mathrm{obm}$ thermometer and a $1 \mathrm{ohm}$ bridge-wire.

A recorder of this type is more expensive than the thread recorder, but is complete in itself, with the exception of the battery, and does not require either an auxiliary resistance box, or a rheostat for adjusting the scale. It is a great advantage in practice that the scale never requires adjustment, but is always correct to about 1 in 1000 , provided that the bridge-wire is correct and uniform. It is easy to change from one scale to another when required by changing the bridge-wire, which gives a range of 1 to 4 , or by changing the thermometer, which gives a range of 1 to 20, from a fundamental interval of $10 \mathrm{ohms}$ to a fundamental interval of $0.5 \mathrm{ohm}$.

For ordinary work thermometers are generally provided with an "ice-bobbin" or balancing coil, equal in resistance to the thermometer at $0^{\circ} \mathrm{C}$. The ice-bobbin for each thermometer is connected to its appropriate terminals when the thermometer to which it belongs is in use. If the thermometer is required to cover an extensive range of temperature, a series of auxiliary resistances, generally ranging from 0 to $20 \mathrm{ohms}$ is provided, which enables the range to be extended to 20 times the range of the $1 \mathrm{ohm}$ bridge-wire. With a $26 \mathrm{ohm}$ thermometer the range thus obtained would be $200^{\circ} \mathrm{C}$., or $2000^{\circ} \mathrm{C}$. with a $2 \cdot 6 \mathrm{ohm}$ pyrometer. For clinical work these auxiliary resistances may be dispensed with if 
the balancing resistance is adjusted to $30 \mathrm{ohms}$ to balance the thermometer at $40^{\circ} \mathrm{C}$, and the zero fixed at the centre of the bridge-wire so as to give a range of from $35^{\circ}$ to $45^{\circ} \mathrm{C}$., with a $1 \mathrm{ohm}$ bridge-wire.

Some observers have experienced trouble with this type of recorder owing to the heating effect of the measuring current. Such troubles have arisen chiefly from the employment of unsuitable thermometers with excessive currents. With a $26 \mathrm{ohm}$ thermometer the instrument can be adjusted to work perfectly on a scile of $4 \mathrm{~cm}$. to $1^{\circ} \mathrm{C}$. with a current of one hundredth of an ampere through the thermometer. The record can be read easily to less than a hundredth of a degree, or 1 in 30,000 ; and the heating effect of the current, with a celluloid thermometer of the surface type above described, is only two or three thousandths of a degree, or practically inappreciable on the most open scale of the record.

Testing and Adjustment of Slide-wire Recorder.

11. The most important points to test in a slide-wire recorder are the adjustment of the zero of the galvanometer, and the zero of the slide-wire. When the galvanometer coil is free and the instrument levelled, the two prongs of the contact fork should be just clear of the contact wheel on either side. If there is any torsion in the suspension, one of the prongs will bear against the wheel, and the pen will travel continuously in one direction when the circuit of the relay magnets is made, the galvanometer circuit being open. The torsion head should be adjusted until the pen does not travel either way under these conditions. This is a very delicate test. If there is much torsion in the suspension, the apparent zero on the slide-wire will vary to some extent with the resistance in circuit when the galvanometer circuit is closed. 'This adjustment is made before the instrument is sent out, but it is desirable to test it occasionally, especially if the suspension has been strained or damaged, or if a new suspension is fitted.

To adjust the zero of the slide-wire, short circuit the pyrometer and compensator terminals, $\mathrm{PP}$ and $\mathrm{CC}$, with short equal pieces of copper wire. When the battery is switched on the pen will quickly come to rest at the zero. 
If the position is not quite correct in reference to the record sheet, the slide-wire may be shifted in the direction of its length through a distance equal to the observed deviation. For many purposes it is most convenient to have the zero in the centre of the slide-wire. The wire can then be changed, if desired, for another of different resistance and scale, without altering the zero. If it is desired to have the zero at one end of the scale, it is necessary to provide a small auxiliary slide for correcting the adjustment when the slidewire is changed. The freedom of the galvanometer suspension from torsion may converiently be tested at the same time as the zero of the slide-wire, by donbling the resistance in the battery circuit, so as to halve the current through the slide-wire. If this does not produce any appreciable shift of zero, the suspension must be very nearly free from torsion. It is important that the external resistance added should be only in the bridge circuit, and not in that of the relay magnets, which will not work satisfactorily if the P.D. on the relay circuit is much reduced. This mistake has often been made in using this type of recorder.

\section{Slide-wire Records.}

12. It is possible to obtain records on a more open scale with the slide-wire recorder than with the thread recorder, owing to the greater width of the record sheet, which is $20 \mathrm{~cm}$. for the slide-wire recorder, as compared with $8 \mathrm{~cm}$. for the

Fig. 12.

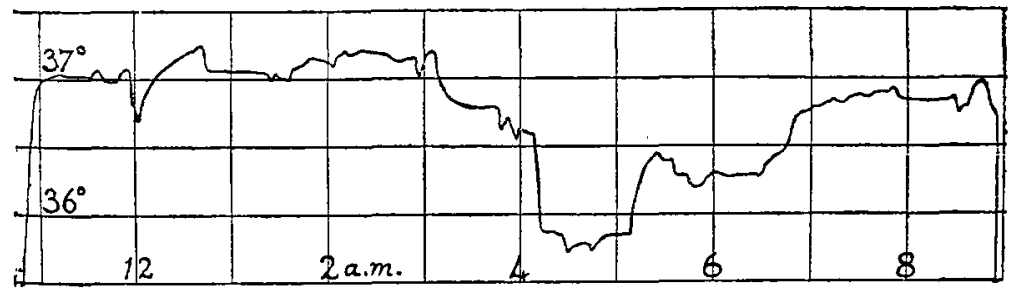

Axillary Thermometer D, on Slide-wire Recorder, $4 \mathrm{~cm} . / \mathrm{deg}$.

thread recorder. The latter is limited by the range of the galvanometer boom, which cannot be indefinitely elongated without making the deflexion too sluggish. The record reproduced in fig. 12 was taken with an axillary thermo- 
meter differing in a few details of construction only from those employed for records 8 and 9 . The scale of the original record was $4 \mathrm{~cm}$. per $1^{\circ} \mathrm{C}$., which is 2.5 times the scale adopted with the thread recorder. The scale has been reduced to one third, to facilitate reproduction and comparison. It will be observed that the record shows the same characteristic dip, culminating between 4 and 5 A.M, as records 8 and 9. When first observed, it seemed as though this dip of temperature might be due to some instrumental error, but it has been observed on several occasions with

Fig. 13.

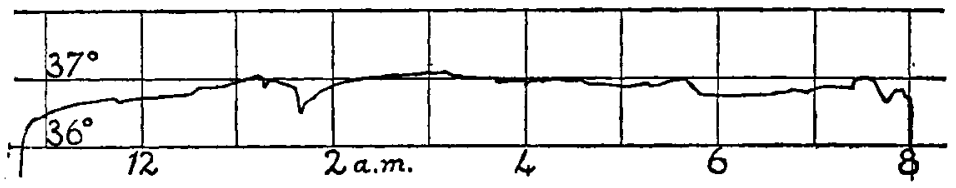

Axillary Thermometer D, on Slide-wire Recorder, $2 \mathrm{~cm}$./deg.

this particular patient, with different thermometers and different recording instruments. The dip is not always present. The record reproduced in fig. 13, which shows an extremely steady temperature, was taken on the same patient, with the same recorder and thermometer, but on a scale of $2 \mathrm{~cm}$. per $1^{\circ} \mathrm{C}$., similarly reduced in reproduction. The

Fig. 14.

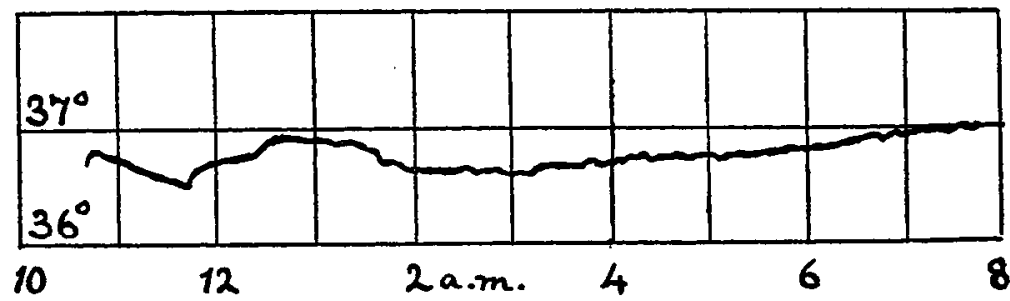

Rectal on Thread Recorder (simultaneous with record 13).

current employed in both cases was the same as with the thread recorder for records 8 and 9 , and the thermometer was attached in precisely the same way. The only effect of reducing the scale with the slide-wire recorder would be to make the instrument proportionately quicker in responding, which cannot account for the greater smoothness of the 
record. A record taken simultaneously on the same patient with the rectal thermometer and thread recorder, is reproduced in fig. 14. The variations shown are very slight, and the differences between the two thermometers are such as would be likely to exist in different parts of the body.

The records above given are sufficient to show, if proof were needed, that accurate clinical records of temperature on a fairly open scale under practical conditions may readily be obtained by the aid of electrical resistance thermometers, with either of the recording instruments above described. The recording instruments are standard types, which have been thoroughly tested by the experience of a great variety of users for many years, and are not.likely to give trouble.

\section{Abstraut.}

The President dealt with the application of resistance thermometers to the recording of clinical temperatures. Records of clinical temperatures have been obtained by Dr. Gangee using a thermocouple in conjunction with sensitive recording instruments. The objections to thermocoupies are twofold. In the first place, the E.M.F.'s developed are so small that the recording instruments must be very sensitive and therefore unsuitable for ordinary use. In the second place, serious difficulties arise with regard to the thermostat which is necessary to maintain one of the junctions of the thermocouple at a constant temperature. The chief difficulty in connexion with the use of resistance thermometers for this lind of work lies in the heating effect of the current. In order to explain how this difficulty is overcome Prof. Callendar gave a short account of the conditions upon which the sensitiveness of a Wheatstone's bridge depends. He pointed out that in platinum thermometry, in order to obtain accurate compensation fur the resistance of the leads, it is necessary that the ratio arms of the bridge should be equal, and he showed that this condition reduced the sensitiveness, which could be obtained by suitably varying the resistances by about 30 per cent. In joining up a bridge in work with resistance thermometers, Maxwell's rule for the positions of the battery and galvanometer which give maximum sensitiveness is seldom applicable. While Maxwell's arrangement actually gives the greatest sensitiveness, the heating effect of the current is so much greater that the trouble arising from this cause more than counterbalances the increased sensitiveness. The problem to be solved in designing a suitable thermometer for clinical work is, with a given galvanometer and resistance-box, to find the resistance of the thermometer which will give the most accurate results for a given heating effect of the current. This is given by the equation $R=2 G+S$, where $G$ is the resistance of the galvanometer and $S$ that of one of the 
ratio arms. In the apparatus used at the meeting $G=S=10$ ohms giving $R=30$ ohms, a resistance for the thermometer which gives the convenient scale of $0.1 \mathrm{ohm}$ increase per degree Centigrade. It is important in the construction of a therwometer for clinical work to secure quickness of action and to reduce the heating effect of the current. An ordinary tube-form of thermometer is good for laboratory work with sensitive galvanometers, hut it is unsuitable for use with recorders. An ordinary form of thermometer takes about $1 \frac{1}{2}$ minutes to reach a steady temperature when taken from water at the temperature of the room and placed in water at $0^{\circ} \mathrm{C}$., whereas a thermometer suitably designed will take up a steady temperature in about ten seconds. The pattern of the thermometer must also be suited to the purpose for which it is intended. Three types were shown, designed for mouth, rectal, and surface work. Experiments were performed proring that these thermometers fulfilled the necessary conditions. The recording instruments for use with these thermometers were briefly described. Prof. Callendar then showed continuous records obtained from a patient with a normal temperature. The temperature is generally very steady if the thermometer does not shift or the patient get wholly or partly out of bed. The effects of external changes of temperature were also shown, and simultaneous records taken on different parts of the body illustrated the fact that the temperature does not vary in the same way at all places. On the medical side there is a vast field for research which may eventually result in important additions to knowledge.

\section{Telephone Circuits. By Professor John Perry, F.R.S.*}

Aвout a year ago Mr. Sidney Brown told me that he wished to place contrivances at equal distances in telegraphic or telephonic circuits and that it was necessary to make calculations, beforehand, because in a telephone line, say, of three hundred miles or more with similar contrivances inserted every one or two miles, it was practically impossible to proceed by any kind of experimental adjustment. He spoke of various kinds of contrivance involving combinations of condensers and inductance coils and transformers and even rotary motors and generators; some in series with the line and others as shunts to earth.

Exact investigation of what occurs in telephonic and telegraphic signalling in general is quite impossible. Trans-

* Read February 25, 1910. 